\title{
Multipath Interference Suppression Technology Based on CSS-VTRM-OFDM
}

\author{
Tao Huating 1 , 2 \\ 1 Wuhan University of Technology, Wuhan China \\ 2 Henan University of Engineering, Zhengzhou, China \\ taohuating@126.com
}

Key words: dense multipath interference, multi carrier modulation, linear spread spectrum, virtual time reversal channel, interference resisting strategy

\begin{abstract}
Multipath interference is the main factor of wireless communication, especially in the dense multipath channel. How to improve the performance of the tunnel wireless communication is a hot issue. From the modulated carrier, spreading, multipath propagation, to de-spreading process gives theoretical analysis and signal description, including OFDM system in frequency selective fading, chirp spread spectrum signal after multipath channel produced frequency shift, and virtual time reversal mirror technology of balancing mechanism. On the basis of this, the strategy of multipath interference suppression in wireless communication is proposed. Simulation results show that the proposed combination strategy has significant effect on improving the quality of communication and transmission performance.
\end{abstract}

\section{OVERVIEW}

In the process of wireless signal propagation, due to the different signal radiation direction, the different components of the signal will have a experience of different paths and different reflection times before arriving at the receiving end. Therefore, the signal at the receiving end will be a superposition of the same frequency non - phase signal components with different degrees of delay and different degrees of fading. This phenomenon leads to the delay spread of the signal, the inter symbol interference and frequency selective fading, which is the multipath effect ${ }^{[1]}$.

Different paths lead to different time delay, which is the main factor of delay spread and inter symbol interference. The superposition of the same frequency and different phase is the main reason for the frequency selective fading. In shape as well as closed tunnels, culverts, mine roadway, a dense multipath environment, propagation of spatial structure will enable suppress multipath interference strategy more challenges and special, and this environment, the interference of multipath effect sometimes severe enough to make the wireless communication can not be effectively achieved ${ }^{[2]}$.

In this paper, we mainly analyze the anti multipath interference characteristics of OFDM system, the frequency shift characteristics of Chirp spread spectrum signal after multipath propagation ${ }^{[3]}$, and the multipath channel equalization mechanism of virtual time reversal technology ${ }^{[4]}$. On the basis of this, the multipath interference suppression combination strategy is studied ${ }^{[5]}$.

\section{DELAY AND FREQUENCY SHIFT OF CHIRP SPREAD SPECTRUM SIGNAL IN MULTIPATH CHANNEL}

Assuming that the traditional OFDM system has $\mathrm{N}$ sub carriers, the sub carrier frequency is $\mathrm{B}$. A is a series of data to be sent, through the serial to parallel conversion, and then be modulated to the $\mathrm{N}$ system of OFDM sub carrier. Assuming that the OFDM symbol is T, the signal can be expressed as:

$$
s(t)=\sum_{k=0}^{N_{k}-1} d_{k} e^{j 2 \pi f_{k} t} R_{T}(t)
$$

Where $d_{k}$ is the data will be sent and which is modulated $R_{T}(t)$ on $k^{\text {th }}$ subcarries, $f_{k}$ is the frequency of $k^{\text {th }}$ subscarries. is a rectangular signal with a period of T. It is assumed that the signal can be 
transmitted in a closed tunnel, a signal with ${ }^{N_{p}}$ path can reach the receiving end, and the channel impulse response can be expressed as:

$h(t)=\sum_{p=0}^{N_{p}-1} a_{p} h_{p} \delta\left(t-\tau_{p}\right)$

Where, $a_{p}$ is the amplitude of path $p^{t h}, \delta\left(t-\tau_{p}\right)$ and $\tau_{p}$, respectively corresponding to the waveform and the time delay of path $p^{\text {th }}$ signal. After transmitted through the dense multipath channel, the signal is reflected by the wall many times will arrive at the receiving end at different time because of experience of the different distance and time delay. Received signal can be expressed as:

$r(t)=s(t) * h(t)+n(t)=\sum_{p=0}^{N_{p}-1} h_{p} s\left(t-\tau_{p}\right)+n(t)$

Formula (3) is the convolution form of signal and channel impulse response, $n(t)$ is the additive noise.The formula (1) and (2) into (3), the received signal can be expressed as:

$r(t)=\sum_{p=0}^{N_{p}-1 N_{k=0}-1} h_{p} e^{-j 2 \pi f_{k} \tau_{p}} d_{k} e^{j 2 \pi f_{k} t} R_{T}(t)+n(t)$

Contrast formula (1) and (4),The influence of channel to The received signal is represented as $p_{k}$

$p_{k}=\sum_{p=0}^{N_{p}-1} h_{p} e^{-j 2 \pi f_{k} \tau_{p}}$

According to the formula (5), it is known that the different components of the same signal experienced different time delay after the multipath channel. At the receiving end, the corresponding signal is superimposed in the same frequency and phase, which is the essential cause of the frequency selective fading. When the $k^{\text {th }}$ carrier is in the deep fading, $p_{k}$ tends to 0 , the data carried on the carrier will be destroyed, and can not be compensated by the use of channel equalization technology.In order to make up for the defects of fixed frequency carrier wireless communication, the carrier signal is processed by linear spread spectrum, and the spread spectrum signal is adopted,

$c_{\text {up }}(t)=e^{j \pi \alpha t^{2}} R_{T}(t)$

where, $\alpha$ is the frequency modulation slope, after linear spread spectrum which is expressed as:

$s_{c}(t)=s(t) \cdot c_{u p}(t)=\sum_{k=0}^{N_{k}-1} d_{k} e^{j 2 \pi \pi_{k} t+j \pi a t^{2}} R_{T}(t)$

Time frequency characteristics of the multi carrier signal before and after the linear spread spectrum is shown in figure 1. Figure (a) represents the time-frequency characteristics of the original multi carrier signal. And the Figure (b) represents is after a linear spread spectrum.

After the multipath channel is represented by (2), the signal expressed by the formula (6) is converted to the signal at the receiving end. As follows:

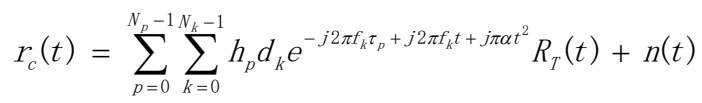
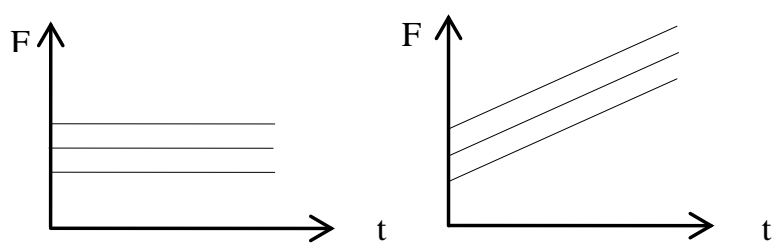

(a) before linear spread spectrum （b)After linear spread spectrum Fig. 1 the schematic diagram before and after chirp spreading

On the receiving end, de-spreading signal is as follows:

$c_{\text {down }}(t)=e^{-j \pi \alpha t^{2}} R_{\mathrm{T}}(t)$

the process of de-spreading of the received signal can be expressed as follows:

$r(t)=r_{c}(t) \cdot c_{\text {down }}(t)+n(t)=\sum_{p=0}^{N_{p}-1} h_{p} S_{c}\left(t-\tau_{p}\right) \cdot e^{-j \pi \alpha t^{2}} R_{T}(t)+n(t)$

$=\sum_{p=0}^{N_{p}-1} \sum_{k=0}^{N_{k}-1} h_{p} d_{k} e^{j 2 \pi f_{k}\left(t-\tau_{p}\right)+j \pi \alpha\left(t-\tau_{p}\right)^{2}-j \pi \alpha t^{2}} R(t)+n(t)=\sum_{p=1}^{N_{p}-1} \sum_{k=0}^{N_{k}-1} h_{p} e^{-j 2 \pi f_{k} \tau_{p}+j \pi \alpha \tau_{p}{ }^{2}-j 2 \pi \alpha \tau_{\rho} t} d_{k} e^{j 2 \pi f_{k} t} R_{T}(t)+n(t)$

$=p_{k}(t) \cdot s(t)+n(t)$ 
Where,

$p_{k}(t)=\sum_{p=0}^{N_{p}-1} h_{p} e^{-j 2 \pi \pi_{k} \tau_{p}+j \pi \alpha \tau_{p}^{2}-j 2 \pi \alpha \tau_{p} t}$

In the formula (11), $e^{-j 2 \pi \alpha \tau_{p} t}$ is a function of time. It shows that after de-spreading, the signal with different time delay gets a different frequency shift, the size of the frequency shift is positive proportional relationship with the chirp rate and time delay of signal. shown in Figure 2

Thus, the different component of homologous signal through the multipath channel arrived at the receiving end is shifted to a different frequency point. This characteristic, on the one hand, ensures that the multipath component does not form a superposition of the same frequency and different phase, thereby avoiding the frequency selective fading. The observed signal frequency at the

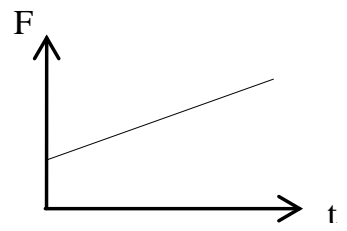

(a) before de-spreading Fig. 2 the schematic diagram of Time-frequency characteristic of signal before and after de-spreading
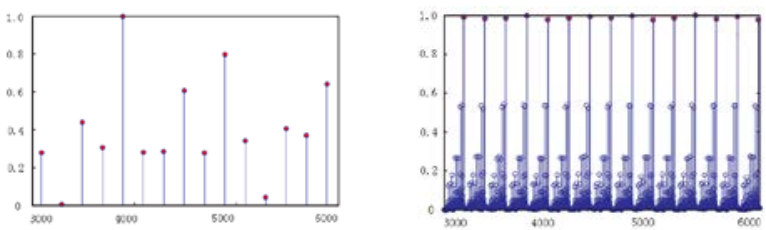

$\begin{array}{ll}\text { (a) the case without chirp spread spectrum } & \text { (b) the case after chirp spread spectrum }\end{array}$ Fig. 3 the schematic diagram of Time-frequency characteristic of signal before and after de-spreading receiver is shown in Figure 3.

\section{VIRTUAL TIME REVERSAL EQUALIZATION TECHNIQUE FOR CSS SPREAD SPECTRUM TRANSMISSION SYSTEM}

Due to the multipath signal energy is not fully utilized, it is not conducive to improve the transmission performance of the system. In order to improve the system transmission performance, VTRM (Time Reversal Mirror Virtual) technology is introduced. VTRM achieves spatial focusing and channel equalization, the delay spread of the multipath diversity is compressed, the multipath energy is focused, and the SNR is effectively improved. In this paper, VTRM and CSS are combined to solve the problem of interference suppression in dense multipath channel. the principle as shown in Figure 4.

First, a detection signal $p(t)$ is transmitted, and then, at the receiving end, the estimated value $h^{\prime}(t)$ of the impulse response of the channel is obtained according to the observed signal $p_{r}(t) . h^{\prime}(t)$ to do the time reversal operation was $h^{\prime}(-t)$, and then convolution
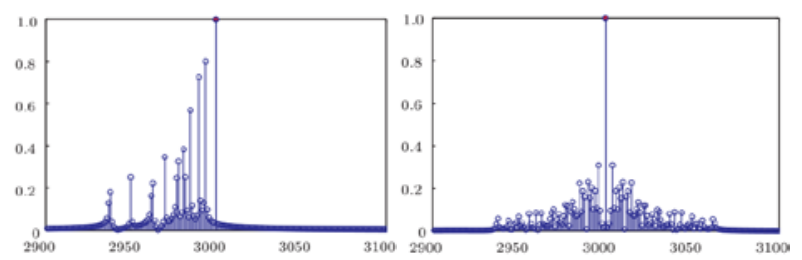

Fig. 4 Schematic diagram of virtual time reversal is done with $h^{\prime}(-t)$ and the received signal,

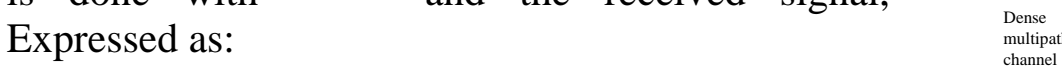
$r(t)=s_{r}(t) * h^{\prime}(-t)=[s(t) * h(t)+n(t)] * h^{\prime}(-t)=s(t) *\left[h(t) * h^{\prime}(-t)\right]+n(t) * h$ $=s(t) * v(t)+n^{\prime}(t)$

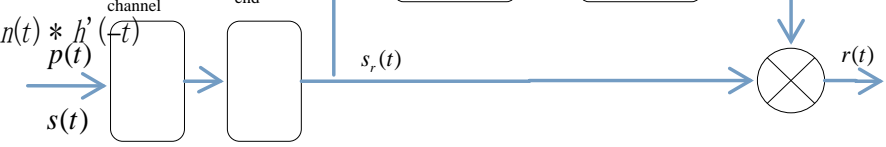

$v(t)=h(t) * h^{\prime}(-t)$, in the formula, is called the virtual time reversal channei. When the impuise (a) The case of time reversal processing (b) After the time conversion processing situation Fig. 5 Sketch map of time reversal processing effect

response of the estimated value, ${ }^{h}(t)$, approaching the actual channel impulse response $h(t), v(t)$ is close to the impulse response function of the autocorrelation. At this time, the peak will be significantly higher than the side. This process has the effect of time and space focusing, and the energy of the multipath channel can be added. As shown in Figure 5, (a) is the case without the time reversal processing, and (b) is the case after the time conversion processing. 


\section{OFDM SYSTEM BASED ON CSS-VTRM}

Virtual time reversal mirror algorithm is applied in css-ofdm system. At the receiving end, the OFDM symbols as (13) (14) shown. $p_{k}(t)$ is the effect of multipath channel on signal. In order to obtain the original transmission signal, do the time reversal processing for $p_{k}(t)$, and then with the formula (14) do convolution operation. As shown below:

$$
r_{0}(t)=\sum_{k=0}^{N-1} p_{k}(t) * p_{k}(-t) d_{k} e^{j 2 \pi f_{k} t} R(t)=\sum_{k=0}^{N-1} v_{k}(t) d_{k} e^{j 2 \pi f_{k} t} R(t)
$$

where,

$V_{k}(t)=p_{k}(t) * p_{k}(-t)=\sum_{p=0}^{N_{p}-1} h_{p}^{2}+\sum_{p=0}^{N_{p}-1} \sum_{\substack{q=0 \\ q \neq p}}^{N_{p}-1} h_{p} e^{-j 2 \pi f_{k}\left(\tau_{p}-\tau_{q}\right)+j \pi \alpha\left(\tau_{p}-\tau_{q}\right)^{2}-j 2 \pi \alpha\left(\tau_{p}-\tau_{q}\right) t}$

Formula (14) is the virtual time reversal channel. The virtual time reversal channel can be obtained by estimating the impulse response of the channel according to the detection signal.OFDM system model based on linear spread spectrum and virtual time reversal mirror technology, as shown in figure 6 .

\section{SIMULATION}

In view of the multipath interference of tunnel, this
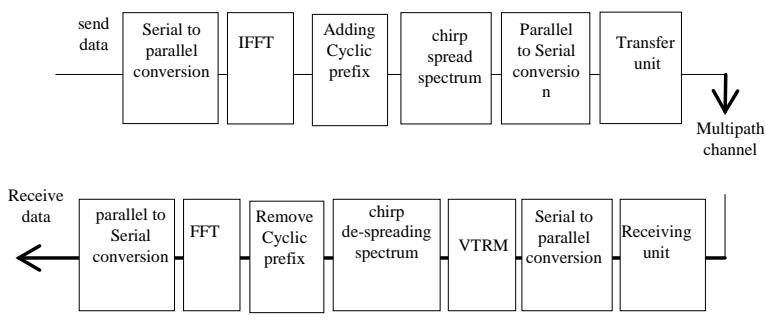

Fig. 6 Block diagram of CSS-VTRM-OFDM system paper proposes a combination Combination strategies include orthogonal frequency division multiplexing based multicarrier transmission, linear spread spectrum and virtual time reversal technology. The theoretical analysis and Simulation of the proposed strategy is completed. The simulation results show that the CSS-VTRM-OFDM system has better BER performance, as shown in figure 7.

\section{REFERENCES}

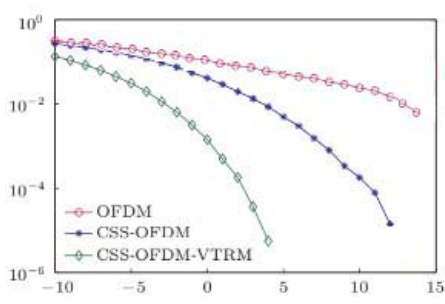

Fig. 7 Sketch map of time reversal processing effect

[1] Tran L N, Hong E K. "Unique Word Based Frequency Domain Equalization with Noise Prediction” .[J]. IEEE Communications Letters, 2008, 12(10):785 - 787.

[2] Stojanovic M, Preisig J. "Underwater acoustic communication channels: Propagation models and statistical characterization” [J]. Communications Magazine IEEE, 2009,47(1):84 - 89.

[3] Lin Xiaoyang; Yuan Fei, "Multiuser underwater acoustic communication based on multicarriermultiple chirp rate shift keying” , OCEANS 2014 - TAIPEI, 7-10 April 2014, ISBN:978-14799-3645-8, IEEE

[4] SAKTHIVEL M S, NATARAJAN V. "Performance analysis of signal to noise ratio and bit error rate for multiuser using passive time rever- sal technique in underwater communication" [A]. Wireless Communication and Sensor Computing[C]. Chennai, 2010.1-4.

[5] Wang Jianwei, Wang Dong, hao Yuping. "Multipath combining scheme for ISI suppression in DS-UWB system”, international Conference on Wireless Communication Networking and mobile Computing Wuhan, 2006:1-4p.

LIU M, CRUSSIERE M, HELARD J F,et al. "A combined time and frequency algorithm for improved channel estimation in TDS-OFDM ” [C] //IEEE ICC 2010. Cape Town:IEEE Press,2010.

[6] LIU M, CRUSSIERE M, HELARD J F,et al. "A combined time and frequency algorithm for improved channel estimation in TDS-OFDM ” [C] //IEEE ICC 2010. Cape Town:IEEE Press,2010.

[7] YIN Yan-ling QIAO Gang LIU Song-zuo , "Underwater acoustic OFDM channel equalization based on virtual time reversal mirror,Journal on Communications " ,10.11959/j.issn.1000436x.2015011,2015, 36(1) 